\title{
Performance Analysis OF Retinal IMAge BLOOd VeSSEL SEgMENTATION
}

\author{
${ }^{1}$ D.Siva Sundhara Raja, ${ }^{2}$ Dr.S.Vasuki, ${ }^{3}$ D.Rajesh Kumar \\ ${ }^{1,3}$ SACS MAVMM Engineering College, Madurai, India \\ ${ }^{2}$ Velammal College of Engineering \& Technology
}

\begin{abstract}
The retinal image diagnosis is an important methodology for diabetic retinopathy detection and analysis. in this paper, the morphological operations and svm classifier are used to detect and segment the blood vessels from the retinal image. the proposed system consists of three stages-first is preprocessing of retinal image to separate the green channel and second stage is retinal image enhancement and third stage is blood vessel segmentation using morphological operations and svm classifier. the performance of the proposed system is analyzed using publicly available dataset.
\end{abstract}

\section{KEY WORDS}

Blood vessels, classifier, diabetic retinopathy, diagnosis, green channel.

\section{INTRODUCTION}

Retinal images are influenced by all the factors that affect the body vasculature in general. The human eye is a unique region of the human body where the vascular condition can be directly observed. In addition to fovea and optic disc, the blood vessels contributes one of the main features of an retinal fundus image and several of its properties are noticeably affected by worldwide major diseases such as diabetes, hypertension, and arteriosclerosis. Further, certain eye diseases such as choroidal neovascularization [1] and retinal artery occlusion [2] also make changes in the retinal vasculature. As per previous statement, the segmentation of blood vessels in retinal images can be a valuable aid for the detection of diabetic retinopathy and glaucoma diagnosis.

An automated segmentation and inspection of retinal blood vessel features such as diameter, color and tortuosity as well as the optic disc morphology allows ophthalmologist and eye care specialists to perform mass vision screening exams for early detection of retinal diseases and treatment evaluation. This could prevent and reduce vision impairments; age related diseases and many cardiovascular diseases as well as reducing the cost of the screening. Over the past few years, several segmentation techniques have been employed for the segmentation of retinal structures such as blood vessels and optic disc and diseases like lesions in fundus retinal images. However the acquisition of fundus retinal images under different conditions of illumination, resolution and field of view (FOV) and the overlapping tissue in the retina cause a significant degradation to the performance of automated blood vessel and optic disc segmentations. 


\section{LITERATURE SURVEY}

In [1], Xiayu et al. used graph based approach for blood vessel boundary delineation. The widths of the retinal blood vessels are measured and its edges are segmented. The graph is constructed based on the vessels weight. The REVIEW database was used in this work. This paper has some deficiencies, such as the crossing points and branching points are currently not treated individually, and consequently the blood vessel detection points are not clearly indicated.

In [2], Benson et al. proposed line-shape concavity measuring model to remove dark lesions which have an intensity structure different from the line-shaped vessels in a retina. This method achieved $95.67 \%$ of an average accuracy for the blood vessel detection with respect to ground truth images in DRIVE database, while provided $95.56 \%$ of an average accuracy for the blood vessel detection with respect to ground truth images in STARE database.

In [3], Miguel et al. presented multi-scale feature extraction and region growing algorithm for retinal blood vessels segmentation. This implementation allowed a faster processing of these images and was based on a data partitioning.

In [4]-[10], different methods for blood vessel detection and segmentation is presented.

Staal [14] et al. proposed a pixel feature based method that additionally analyzed the vessels as elongated structures. The edge-based methods can be further classified into window-based and tracking-based methods. Window-based method estimates a match at each pixel against the pixel's surrounding window. In order to trace the vessels, the tracking approach makes use of local image properties from an initial point.

\section{PROPOSED SYSTEM}

The proposed system consists of three stages-first is preprocessing of retinal image to separate the green channel and second stage is retinal image enhancement and third stage is blood vessel segmentation using morphological operations and SVM Classifier. The proposed system for blood vessel segmentation is illustrated in Fig. 1.

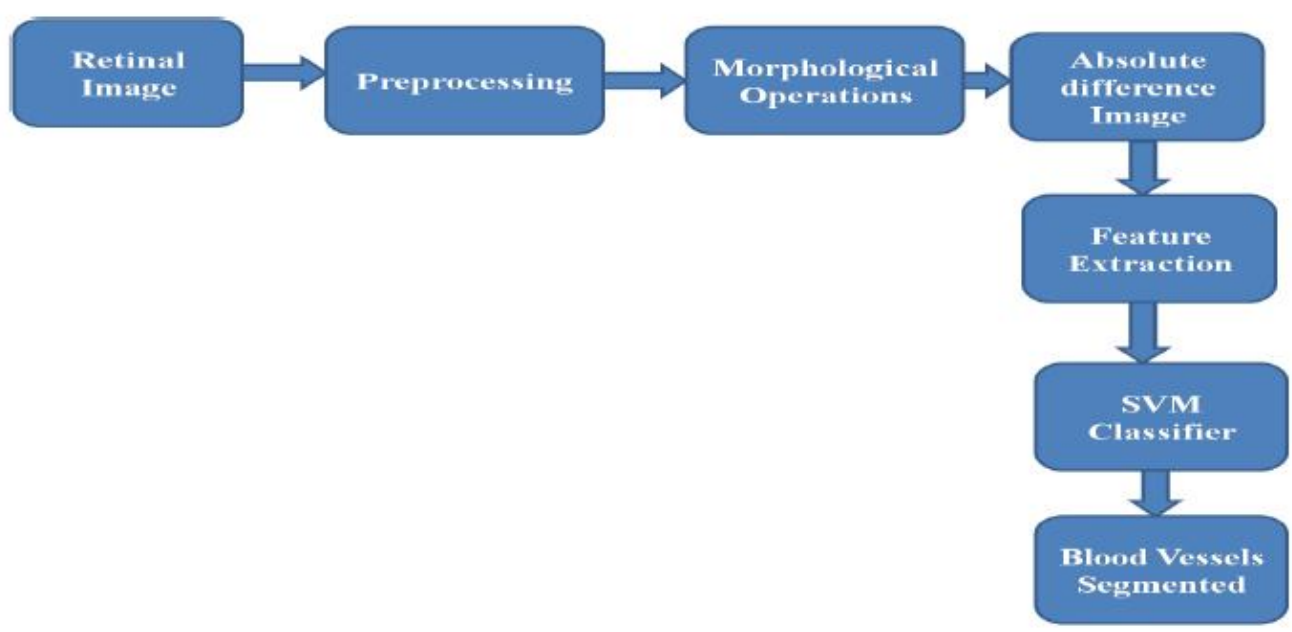

Fig.1. Proposed blood vessel segmentation system. 


\section{A. Preprocessing}

Initially the retinal image is enhanced using adaptive histogram equalization technique in order to enhance the blood vessels. Then, the retinal fundus image is divided in to three primary components such as Red Channel (R), Green Channel (G) and Blue Channel (B). The green channel is high sensitive to the blood vessels. Hence this channel is considered for the detection and segmentation of retinal blood vessels from the retinal image.

\section{B. Morphological Operations}

The morphological operations are applied on the pre processed green channel. Morphological operation processes the preprocessed image with structuring element. The retinal blood vessels are detected by applying dilation and erosion process to a preprocessed image. The morphological opening and closing operation are applied to an image based on multi structure elements to enhance the vessel edges. Morphological opening and closing operation is performed by using dilation and erosion. The morphologically processed opened image and morphologically processed closed images are absolutely subtracted to detect the blood vessels from retina fundus image. The combination of dilation and erosion operations is performed on image with different structuring element of radius 3. Then, an absolute difference mapping image is formed by absolute subtraction of retinal image from the morphologically processed sub-band image.

\section{Feature Extraction and Classifier}

The Local Binary Pattern (ELBP) features and GLCM features are extracted from the morphologically processed image. It can be computed as in Eq. 1,

$$
\mathrm{LBP}=\sum_{p=1}^{8} 2^{p} * K\left(I_{N}-I_{C}\right)
$$

where, IN denotes the neighboring pixel in a square window $(3 \times 3)$, IC is the center pixel in the $3 \times 3$ mask, ' $p$ ' represents the number of surrounding pixels, ' $\mathrm{K}$ ' denotes c function and $\mathrm{K}$ (IN $-\mathrm{IC}$ ) is marked as the threshold value and it is estimated as,

$$
K\left(I_{N}-I_{C}\right)=\left\{\begin{array}{l}
1, \text { if } I_{N}-I_{C} \geq 0 \\
0, \text { if } I_{N}-I_{C}<0
\end{array}\right.
$$

\section{GLCM Features}

The Co-occurrence features can be extracted from each Co-occurrence Matrix. Energy, contrast, correlation and homogeneity are used as GLCM features. 
TABLE I. Feature Representations

\begin{tabular}{|c|c|}
\hline GLCM Features & $\begin{array}{c}\text { Feature } \\
\text { Representations }\end{array}$ \\
\hline 'Contrast' & $\sum|\mathrm{i}-\mathrm{j}|^{\wedge} 2 \cdot \mathrm{p}(\mathrm{I}, \mathrm{j})$ \\
\hline 'Energy' & $\sum \mathrm{p}(\mathrm{I}, \mathrm{j})^{\wedge} 2$ \\
\hline 'Homogeneity' & $\sum \mathrm{p}(\mathrm{I}, \mathrm{j}) /[1+|\mathrm{i}-\mathrm{j}|]$ \\
\hline 'Correlation' & $\begin{array}{c}\sum(\mathrm{i}-\mu \mathrm{i})(\mathrm{j}- \\
\mu \mathrm{j}) \cdot \mathrm{p}(\mathrm{I}, \mathrm{j}) /[\sigma \mathrm{i} . \sigma \mathrm{j}]\end{array}$ \\
\hline
\end{tabular}

The extracted features are trained and the given retinal images are classified in accordance with the trained values SVM Classifier. The SVM classifies each pixel in the retinal image as blood vessel or non blood vessel pixels.

\section{RESULTS AND DISCUSSIONS}

The proposed blood vessel detection and segmentation methodology is applied on images available in DRIVE and STARE databases and the segmentation results were compared with their respective ground truth images.

To measure the performance of the proposed method for the detection of blood vessels on the fundus image, the proposed vessel segmentation method is compared to its corresponding ground truth images. The performance of proposed vessel detected image is experimentally validated with ground truth images.

The performance of proposed blood vessel segmentation methodology is analyzed with the following parameters:

- Sensitivity $(\mathrm{Se}=\mathrm{TP} /(\mathrm{TP}+\mathrm{FN}))$

- Accuracy $(\mathrm{Acc}=(\mathrm{TP}+\mathrm{TN}) /(\mathrm{TP}+\mathrm{FN}+\mathrm{TN}+\mathrm{FP}))$

where, TP denotes true positive, FP denotes false positive, FN denotes false negative and TN denotes true negative. True Positive refers to the correctly detected blood vessels, True Negative refers to the wrongly detected blood vessels, False Positive refers to the correctly and wrongly detected non blood vessel pixels. 
Advanced Computing: An International Journal (ACIJ), Vol.5, No.2/3, May 2014
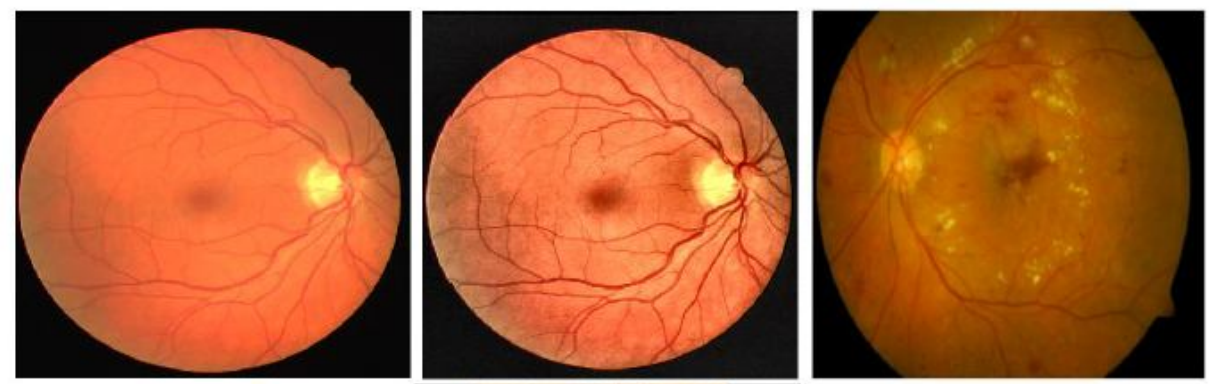

(a)

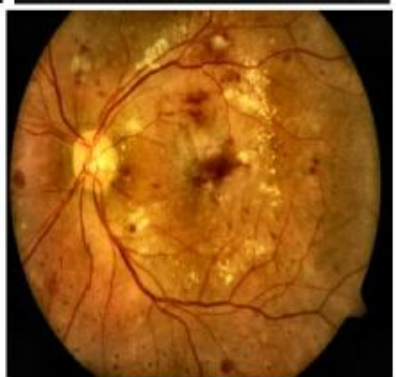

(b)

(c)

(d)

Fig. 2. Retinal image enhancement (a) Normal retinal image (b) Abnormal retinal image (c)-(d) Enhanced retinal image.
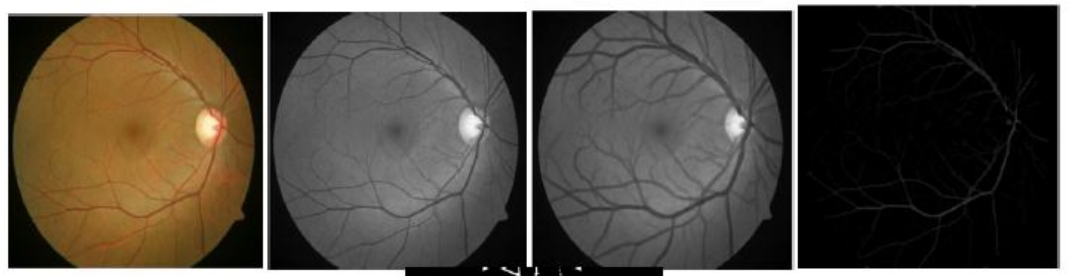

(a)

(b)

(c)

(d)

(e)

Fig. 3. Blood vessel segmentation of normal retinal image (a) Normal retinal image (b) Green channel image (c) Morphologically processed image (d) Absolute difference image (e) Blood vessel segmented image. 

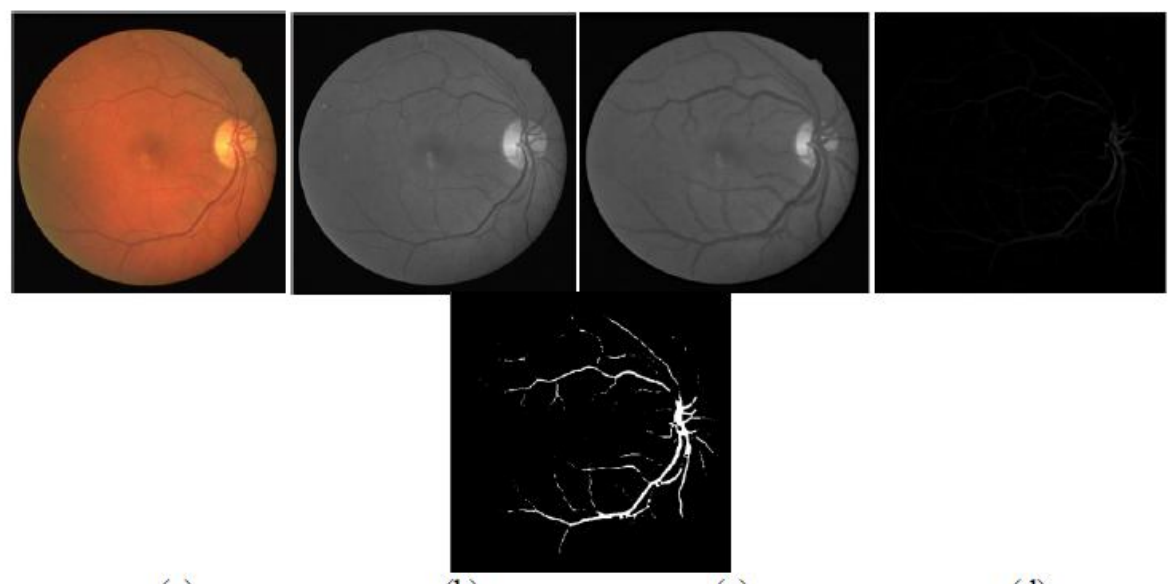

(a)

(b)

(c)

(d)

(e)

Fig. 4. Blood vessel segmentation of abnormal retinal image (a) Abnormal retinal image (b) Green channel image (c) Morphologically processed image (d) Absolute difference image (e) Blood vessel segmented image.

TABLE II. Performance Analysis of Vessel Segmentation

\begin{tabular}{|c|c|c|c|}
\hline Methodology & Se & Sp & Acc \\
\hline Proposed & 0.7800 & 0.9799 & 0.9510 \\
\hline $\begin{array}{c}\text { Soares et al. } \\
{[15]}\end{array}$ & 0.7283 & 0.9788 & 0.9466 \\
\hline $\begin{array}{c}\text { Mendonc et } \\
\text { al. [16] }\end{array}$ & 0.7344 & 0.9764 & 0.9452 \\
\hline
\end{tabular}

The experimental result shows that, Morphological and SVM based blood vessel segmentation achieves a performance appraisal of $78 \%$ sensitivity, $97.99 \%$ specificity, and $95.1 \%$ accuracy, which is clearly shown in Table II.

\section{CONCLUSION}

The blood vessel detection and segmentation is an important for diabetic retinopathy diagnosis at earlier stage. The morphological and SVM classifier is proposed in this paper to detect and segment the blood vessels from the retinal image. The local binary pattern and GLCM features are extracted from the morphologically processed image and used as blood vessels features. The proposed method detected blood vessels with an average sensitivity of $78 \%$, average specificity of $97.99 \%$ and an average accuracy of $99.6 \%$ in the retinal fundus images.

\section{REFERENCES}

[1] X. Xu, M. Niemeijer, Q. Song, M. Sonka, M. K. Garvin, J. M. Reinhardt, and M. D. Abràmoff, - Vessel boundary delineation on fundus images using graph-based approach,\| IEEE Transactions on Medical Imaging, vol. 30, no. 6, June 2011.

[2] B. S. Y. Lam, Y, Gao, and A. W.-C. Liew, - General retinal vessel segmentation using regularization based multiconcavity modeling,\| IEEE Transactions on Medical Imaging, vol. 29, no. 7, July 2010. 
[3] M. A. Palomera-P'erez, M. Elena Martinez-Perez, Hector Ben'itez-P'erez, and Jorge Luis OrtegaArjona, -Parallel multiscale feature extraction and region growing: application in retinal blood vessel detectionll, IEEE Transactions on Information Technology in Biomedicine, vol. 14, no. 2, March 2010.

[4] R. Horaud and T. Skordas, - Stereo correspondence through feature grouping and maximal cliques,\| IEEE Trans. Pattern Anal. Machine Intell, vol. 11, pp. 1168-1180, Nov. 1989.

[5] W. M. Well, - Statistical approaches to feature-based object recognition, Int. J. Comput. Vision, vol. 21, no. 1, pp. 63-98, 1997.

[6] S. Chaudhuri, S. Chatterjee, N. Katz, M. Nelson, and M. Goldbaum, -Detection of blood vessels in retinal images using two-dimensional matched filters,\| IEEE Trans. Med. Imag., vol. 8, pp. 263-269, Sept. 1989.

[7] A. Can, H. Shen, J. N. Turner, H. L. Tanenbaum, and B. Roysam, -Rapid automated tracing and feature extraction from retinal fundus images using direct exploratory algorithms, $\|$ IEEE Trans. Inform. Technol. Biomed., vol. 3, pp. 125-138, June 1999.

[8] L. Gagnon, M. Lalonde, M. Beaulieu, and M.-C. Boucher, -Procedure to detect anatomical structures in optical fundus images,\| in Proc. SPIE Med. Imaging: Image Processing, vol. 4322, 2001, pp. 1218-1225.

[9] S. N. Kalitzin, J. J. Staal, B. M. ter Haar Romeny, and M. A. Viergever, -A computational method for segmenting topological point sets and application to image analysis, IEEE Trans. Pattern Anal. Machine Intell., vol. 23, pp. 447-459, May 2001.

[10] Zana and J.-C. Klein, -Segmentation of vessel-like patterns using mathematical morphology and curvature evaluation,\|l IEEE Trans. Med. Imag., vol. 11, no. 7, pp. 1111-1119, Jul. 2001.

[11] C. Sinthanayothin, J. F. Boyce, H. L. Cook, and T. H.Williamson, -Automated localization of the optic disc, fovea, and retinal blood vessels from digital colour fundus images, $\|$ Br. J. Ophthalmol., vol. 83, pp. 902-911, 1999.

[12] M. Niemeijer and B. van Ginneken, 2002 [Online]. Available: http://www.isi.uu.nl/Reseach/Databases/DRIVE/results.php

[13] A. Hoover, STARE database [Online]. Available: http:// www.ces.clemson.edu/ ahoover/stare

[14] J. Staal, M. D. Abrámoff, M. Niemeijer, M. A. Viergever, and B. van Ginneken, -Ridge-based vessel segmentation in color images of the retina,॥ IEEE Trans. Med. Imag., vol. 23, no. 4, pp. 501509, Apr. 2004.

[15] J. V. B. Soares, J. J. G. Leandro, R. M. Cesar Jr., H. F. Jelinek, and M. J. Cree, -Retinal vessel segmentation using the 2-D Gabor wavelet and supervised classification,\| IEEE Transactions on Medical Imaging, vol. 25, no. 9, pp. 1214-1222, 2006.

[16] A. M. Mendonc, a and A. Campilho, - Segmentation of retinal blood vessels by combining the detection of centerlines and morphological reconstruction,\| IEEE Transactions on Medical Imaging, vol. 25, no. 9, pp. 1200-1213, 2006. 